\title{
GROUP ALGEBRAS OF SOME GENERALISED SOLUBLE GROUPS
}

\author{
by R. P. KNOTT \\ (Received 18th March 1971)
}

1. In (8) Stonehewer referred to the following open question due to Amitsur: If $G$ is a torsion-free group and $F$ any field, is the group algebra, $F G$, of $G$ over $F$ semi-simple? Stonehewer showed the answer was in the affirmative if $G$ is a soluble group. In this paper we show the answer is again in the affirmative if $G$ belongs to a class of generalised soluble groups.

We define $\mathcal{N}$ to be the class of nilpotent groups, $\mathscr{A}$ to be the class of abelian groups. We use $E$ and $L$ to denote the closure operations introduced by $\mathrm{P}$. Hall in (3). Then $E L \mathcal{N}$ denotes the class of all groups having an ascending locally nilpotent series. We recall that in (7) Plotkin has shown that each $E L \mathcal{N}$ group $G$ has an ascending series with $L \mathscr{N}$-factors such that each term is normal in $G$. Since $\mathscr{A} \leqq \mathscr{N}$ and $L \mathscr{A}=\mathscr{A}$, it is clear that $E \mathscr{A} \leqq E L \mathcal{N}$. The former is the class $S N^{*}$ in the notation of Kurosh (4) and has recently been studied by Wallace (10) under the name of restricted $S N$-groups. He has shown that if $G \in E \mathscr{E} \mathscr{A}$ and $F$ is any field of characteristic $p$ where $G$ has no non-trivial elements of order $p$, then $F G$ is semi-simple.

We note that if $G$ is a $S N$-group and $F$ any field of characteristic zero then Villamayor (9) has shown that $F G$ is semi-simple. We have been unable to extend our result to this larger class.

If $G$ is any group, the Hirsch-Plotkin radical of $G$, denoted by $\eta(G)$, is the product of all the normal $L \mathcal{N}$-subgroups of $G$. It is known that $\eta(G)$ is normal and $L \mathcal{N}$.

Let $G$ be any group and $F$ any field. Then $F G$ will denote the group algebra of $G$ over $F$. We denote the Jacobson radical of $F G$ by $J(F G)$ and recall that $F G$ is Jacobson semi-simple if and only if $J(F G)=\{0\}$.

Let $H$ be any subgroup of $G$ and $T$ a right transversal to $H$ in $G$. We will assume that $T$ contains 1 to represent the coset $H$. Let $x$ be any non-zero element of $F G$. Then $x$ can be written uniquely in the form

$$
x=\sum_{i=1}^{n} \alpha_{i} g_{i}
$$

where $n \geqq 1,0 \neq \alpha_{i} \in F H$ and the $g_{i}$ 's are distinct elements of $T$ for $i=1$, $2, \ldots, n$.

The integer $n$ occurring in (1) is called the " $H$-length" of $x$. If $g \in G$ then $x$ and $x g$ have the same $H$-length. Hence, if $g \in T$ and $F G$ has an element of

E.M.S.-A 
$H$-length $n$, there exists an element $x \in F G$ of $H$-length $n$ and $g_{i}=g$ for some $i$ in (1). The remainder of this paper is divided into two sections. In the next section we prove certain lemmas necessary for the proof of our theorem. The proof of this theorem is given in the final section.

The author would like to thank Dr S. E. Stonehewer for suggesting this problem and for his help and encouragement during the preparation of this paper. This work was carried out while the author held a Science Research Council studentship.

2. We begin this section with a lemma due to Amitsur.

Lemma 1. Let $G$ be any group, $H$ any subgroup and $F$ any field, then $J(F G) \cap F H \leqq J(F H)$.

This was first proved in (1).

Lemma 2. Let $F$ be any field and $G$ a torsion-free $L \mathcal{N}$-group. Then the following conclusions hold:

(a) FG has no zero-divisors.

(b) $J(F G)=\{0\}$.

Proof. We recall that if $G$ is a torsion-free $L \mathscr{N}$-group, then $G$ can be ordered (6). The proofs of $(a)$ and (b) can be found in (2).

Lemma 3. Let $G$ be any group such that $\eta(G)$ is torsion-free and greater than 1. Let $F$ be any field such that $F G$ is not semi-simple. Then there exists a subgroup $G_{1}$ of $G$ such that $F G_{1}$ is not semi-simple and the centre of $G_{1}$ is non-trivial.

Proof. If $G$ has a non-trivial centre $G=G_{1}$ will do. If not, choose $0 \neq x \in J(F G)$. Let $H=\eta(G)$ and let $T$ be a transversal (with 1 ) to $H$ in $G$. Then $x$ can be uniquely written in the form

$$
x=\sum_{i=1}^{n} \alpha_{i} g_{i}
$$

where $n \geqq 1,0 \neq \alpha_{i} \in F H$ and the $g_{i}$ 's are distinct elements of $T$ for $1 \leqq i \leqq n$. Suppose that $x$ has minimal $H$-length among the non-zero elements of $J(F G)$. We can assume that one of the $g_{i}$ 's, $g_{1}$ say, is 1 , since $J(F G)$ is an ideal of $F G$.

Now each $\alpha_{i}$ belongs to $F H$ and can be written uniquely in the form

$$
\alpha_{i}=\sum_{h \in \mathbb{H}} \gamma_{h} h,
$$

where $\gamma_{h} \in F$ and all but a finite number are zero. Let supp $\left(\alpha_{i}\right)$ be the set of all $h \in H$ such that $\gamma_{h}$ is non-zero.

We can assume that $\operatorname{supp}\left(\alpha_{i}\right) \neq 1$ for some $i$, since $x$ and $x k$ have the same $H$-length for all $k \in H$.

Let $H(x)=\left\langle\operatorname{supp}\left(\alpha_{i}\right): 1 \leqq i \leqq n\right\rangle$. 
Then $H(x)$ is a finitely generated subgroup of $H$ and hence nilpotent. Also, $H(x)$ is greater than 1 .

Let $Z \neq\langle 1\rangle$ be the centre of $H(x)$. Choose $h \in Z$, then $x-x^{h} \in J(F G)$ where $x^{h}=h^{-1} x h$.

$$
x^{h}=\sum_{i=1}^{n} \alpha_{i}\left[h, g_{i}^{-1}\right] g_{i} \text { and }\left[h, g_{i}^{-1}\right] \in H,
$$

since $H$ is normal in $G$.

$$
x-x^{h}=\sum_{i=2}^{n} \alpha_{i}\left(1-\left[h, g_{i}^{-1}\right]\right) g_{i}=0,
$$

by the choice of $x$. Thus, $\alpha_{i}\left(1-\left[h, g_{i}^{-1}\right]\right)=0$ by uniqueness. $\alpha_{i} \neq 0$ and since $\alpha_{i}\left(1-\left[h, g_{i}^{-1}\right]\right) \in F H$, Lemma 2 implies $\left[h, g_{i}^{-1}\right]=1$ for $1 \leqq i \leqq n$.

Let $G_{1}=\left\langle H(x), g_{i}: 1 \leqq i \leqq n\right\rangle$. Then clearly $Z$ is contained in the centre of $G_{1}$ and $F G_{1}$ is not semi-simple by Lemma 1.

3. In this section we prove the main result of this paper.

Theorem. Let $G$ be any torsion-free ÉL $\mathcal{N}$-group and $F$ any field, then $F G$ is semi-simple.

Proof. Assume that the theorem is false and let $\mathscr{Y}$ be the collection of groups $G$ such that $G$ is a torsion-free $E L \mathscr{E}$-group with a non-trivial centre and $F G$ is not semi-simple. Such a collection is non-empty by Lemma 3. We now find a group $G^{*}$ in $\mathscr{Y}$ such that if $H^{*}=\eta\left(G^{*}\right)$ then $H^{*}$ coincides with the centre of $G^{*}$.

The rest of the proof is as follows:

Let $L / H^{*}$ be the Hirsch-Plotkin radical of $G^{*} / H^{*} . \quad L$ is normal in $G^{*}$ and $L / H^{*}$ is locally nilpotent. However $H^{*}$ is the centre of $G^{*}$ and hence $L$ is locally nilpotent and contained in $\eta(G)$. Thus $H^{*}=L=G^{*}, G^{*}$ is abelian and Lemma 2 gives a contradiction. We have to find $G^{*} \in \mathscr{Y}$ to prove the theorem. Choose $G \in \mathscr{Y}$ and let $H=\eta(G)$. Choose $T$ to be a transversal to $H$ in $G$ where we assume $T$ contains 1 . Then each non-zero $x \in F G$ can be written uniquely in the form

$$
x=\sum_{i=1}^{n} \alpha_{i} g_{i} \text {, where } n \geqq 1,0 \neq \alpha_{i} \in F H
$$

and the $g_{i}$ are distinct elements of $T$ for $1 \leqq i \leqq n$.

Let $N$ be the centre of $G$. Then $N \leqq H$ and we choose $S$ to be a transversal (with 1) to $N$ in $H$. Now each $\alpha_{i}$ can be written uniquely in the form

$$
\alpha_{i}=\sum_{j=1}^{m(i)} \beta_{i, j} h_{i, j}, \text { where } m(i) \geqq 1,0 \neq \beta_{i, j} \in F N
$$

and the $h_{i, j}$ are distinct elements of $S$ for $i$ fixed and $1 \leqq j \leqq m(i)$.

Let $t(x)$ be the minimal $m(i)$ for $1 \leqq i \leqq n$, then $t(x)=m(j)$ for some $j$ and, without loss of generality, we may assume $j=1$. 
Let $r(x)$ be the ordered pair $(n, t(x))$ and choose $x$ from among the non-zero elements of $J(F G)$ such that $x$ is minimal with respect to $\preccurlyeq$ the lexicographic ordering on $Z^{+} \times Z^{+}$.

We define $r(x)=r(G)$ and choose $G \in \mathscr{Y}$ such that $r(G)$ is minimal with respect to $\preccurlyeq$ among the elements of $\mathscr{Y}$. For this $G$ choose $x \in J(F G)$ such that $r(x)=r(G)$ and, in the decomposition of $x, g_{1}=h_{1,1}=1$. This we can do since $r(x)=r(x g)$ for all $g \in G$.

Let $G^{*}=\left\langle N, h_{i, j} g_{i}: 1 \leqq j \leqq m(i), 1 \leqq i \leqq n\right\rangle$. Then $N \leqq N^{*}$, the centre of $G^{*}$ and $H \cap G^{*} \leqq H^{*}=\eta\left(G^{*}\right)$. By Lemma $1, x \in J\left(F G^{*}\right)$ and hence $G^{*} \in \mathscr{Y}$.

Hence we have to show $H^{*}=N^{*}$ to complete the proof. $h_{i, j} g_{i}$ and $h_{i, k} g_{i}$ belong to the same coset of $H^{*}$ in $G^{*}$. Let this coset be $H^{*} g_{i}^{*}$ where $g_{i}^{*}$ belongs to some transversal $T^{*}$ to $H^{*}$ in $G^{*}$. We assume $T^{*}$ contains 1 and $g_{1}^{*}=1$.

Then each $h_{i, j} g_{i}$ can be written in the form $h_{i, j} g_{i}=n_{i, j}^{*} h_{i, j}^{*} g_{i}^{*}$ where $n_{i, j}^{*} \in N^{*}$, and $h_{i, j}^{*}$ belongs to some transversal to $N^{*}$ in $H^{*}$. We can assume $h_{1,1}^{*}=1$.

Thus $x$ can be written in the form $x=\sum_{i=1}^{n} \sum_{j=1}^{m^{*}(i)} \beta_{i, j}^{*} h_{i, j}^{*} g_{i}^{*}$ where $\beta_{i, j}^{*}=\beta_{i, j} n_{i, j}^{*}$ and, for fixed $i$, the $h_{i, j}^{*}$ are distinct. By the choice of $n$ the $g_{i}^{*}$ 's are distinct and, by the choice of $x, m(1)=m^{*}(1) \leqq m^{*}(i) \leqq m(i)$.

Let $B$ denote the subgroup $B=\left\langle N^{*}, h_{i, k}^{*}: 1 \leqq k \leqq m(i), 1 \leqq i \leqq n\right\rangle$. Then $B \leqq H^{*}$ and $B / N^{*}$ is finitely generated. Hence $B / N^{*}$ is nilpotent and since $N^{*}$ is the centre of $G^{*}, B$ is nilpotent. Also $N^{*} \leqq M$, where $M$ is the centre of $B$.

Let $\alpha_{i}^{*}=\sum_{j=1}^{m^{*}(i)} \beta_{i, j}^{*} h_{i, j}^{*}$. Choose $g \in M$ and let $x^{g}$ denote $g^{-1} x g$. Since $J\left(F G^{*}\right)$ is a two-sided ideal of $F G^{*}, x-x^{g}$ belongs to $J\left(F G^{*}\right)$. However, $x^{g}=\sum_{i=1}^{n} \alpha_{i}^{*}\left[g, g_{i}^{*-1}\right] g_{i}^{*}$, where $\left[g, g_{i}^{*-1}\right] \in H^{*}$ for each $i$, since $H^{*}$ is normal in $G^{*}$. Thus, $x-x^{g}=\sum_{i=2}^{n} \alpha_{i}\left(1-\left[g, g_{i}^{*-1}\right]\right) g_{i}^{*}$, since $g_{1}^{*}=1$. By the choice of $x, x-x^{g}=0$ and hence by uniqueness, $\alpha_{i}^{*}\left(1-\left[g, g_{i}^{*-1}\right]\right)=0$ for all $i$. However $H^{*}$ is a locally nilpotent torsion-free group and $\alpha_{i}^{*}$ is non-zero for each $i, 1 \leqq i \leqq n$. Therefore Lemma $2(a)$ implies that $g_{i}^{*}=\left(g_{i}^{*}\right)^{g}$ for $i=2,3, \ldots n$ and all $g \in M$. Thus, $M=N^{*}$.

Let $K / M$ be the centre of $B / M$ and choose $k \in K$. Then $x-x^{k} \in J\left(F G^{*}\right)$,

$$
\begin{aligned}
x^{k}=\beta_{1,1}^{*} & +\sum_{j=2}^{m *(1)} \beta_{1, j}^{*}\left[k, h_{1, j}^{*-1}\right] h_{1, j}^{*} \\
+ & \sum_{i=2}^{n}\left(\alpha_{i}^{*}\right)^{k}\left[k, g_{i}^{*-1}\right] g_{i}^{*},
\end{aligned}
$$


where $\left[k, h_{i, j}^{*-1}\right] \in N^{*}$ and $\left(\alpha_{i}^{*}\right)^{k}\left[k, g_{i}^{*-1}\right] \in F H^{*}$. Whence

$$
x-x^{k}=\sum_{j=2}^{m^{*}(1)} \beta_{1, j}^{*}\left(1-\left[k, h_{1, j}^{*-1}\right]\right) h_{1, j}^{*}+\sum_{i=2}^{n}\left(\alpha_{i}^{*}-\left(\alpha_{i}^{*}\right)^{k}\left[k, g_{i}^{*-1}\right]\right) g_{i}^{*} .
$$

Thus by the choice of $x, x-x^{k}=0$. By uniqueness $\alpha_{i}^{*}-\left(\alpha_{i}^{*}\right)^{k}\left[k, g_{i}^{*-1}\right]=0$. Hence it follows that $\alpha_{i}^{*} g_{i}^{*}=\left(\alpha_{i}^{*} g_{i}^{*}\right)^{k}$ for $1 \leqq i \leqq n$ and all $k \in K$.

Thus the number of conjugates of each $h_{i, j}^{*} g_{i}^{*}$ under transformation by elements of $K$ cannot exceed $\left|\operatorname{supp}\left(\alpha_{i}^{*}\right)\right|$. Thus $K / K \cap N^{*}=K / M$ is finite. However, $B$ is nilpotent torsion-free and thus by (5) each factor of the upper central series of $B$ is torsion-free. Thus, $K / M$ is torsion-free and thus $K=M=B$. Thus each of the $\alpha_{i}^{*}$ 's belongs to $F N^{*}$. Choose $h$ to be any element of $H^{*}$. Then $x-x^{h} \in J\left(F G^{*}\right)$ for $x \in J\left(F G^{*}\right) . \quad x^{h}=\sum_{i=1}^{n} \alpha_{i}^{*}\left[h, g_{i}^{*-1}\right] g_{i}^{*}$, where $\left[h, g_{i}^{*-1}\right] \in H^{*}$. Hence $x-x^{h}=\sum_{i=2}^{n} \alpha_{i}^{*}\left(1-\left[h, g_{i}^{*-1}\right]\right) g_{i}^{*}=0$ by the choice of $n$. It follows from uniqueness that for $i=2,3, \ldots, n, \alpha_{i}^{*}\left(1-\left[h, g_{i}^{*-1}\right]\right)=0$. By Lemma 2(a), $g_{i}^{*}=\left(g_{i}^{*}\right)^{h}$ since $\alpha_{i}^{*} \neq 0$. Thus $h \in N^{*}$ and hence $H^{*}=N^{*}$ and we have found our $G^{*}$.

\section{REFERENCES}

(1) S. A. Amrrsur, On the semi-simplicity of group algebras, Michigan Math. J. 6 (1959), 251-253.

(2) A. A. BovDI, Group rings of torsion-free groups, Sibirsk. Mat. Z. 1 (1960), 555-558.

(3) P. Hall, On non-strictly simple groups, Proc. Cambridge Phil. Soc. 59 (1963), 531-553.

(4) A. G. KURosh, The Theory of Groups (Chelsea, New York, 1960).

(5) A. I. MAL'Cev, Nilpotent torsion-free groups, Izv. Akad. Nauk. USSR Ser. Mat. 13 (1949), 201-212.

(6) B. H. Neumann, On ordered groups, Amer. J. Math. 71 (1949), 1-18.

(7) B. I. PLotkIN, Radical groups, Mat. Sb. N. S. 37 (79) (1955), 507-526: Transl. Amer. Math. Soc. (2) 17 (1961), 9-28.

(8) S. E. STONEHEWER, Group algebras of some torsion-free groups, J. Algebra 13 (1969), 143-147.

(9) O. E. Villamayor, On the semi-simplicity of group algebras II, Proc. Amer. Math. Soc. 10 (1959), 27-31.

(10) D. A. R. WALLACE, The radical of the group algebra of a subgroup, of a polycyclic group and of a restricted SN-group, Proc. Edinburgh Math. Soc. 17 (II) (1970), 165-171.

DePARTMENT OF MATHEMatics

ShefField POLYTECHNiC 